is that teleology is exactly what Lovelock had to purge from his original Gaia hypothesis to satisfy his scientific critics. They may well squirm at the sight of "Gaia's goal" returning.

The reason Gaia needs our help, Lovelock argues, is that the unconscious negative-feedback mechanism that has so far kept the planet cool is destined to fail. Up to now, the Sun's brightening, which tends to warm Earth, has been counteracted by accelerated weathering of the continents, which has decreased $\mathrm{CO}_{2}$ levels in the atmosphere. But before we started boosting it, the $\mathrm{CO}_{2}$ concentration was getting close to the lower limit for photosynthesis. Hence there is a need for either some other cooling mechanism, or a more heattolerant intelligent life form.

Lovelock tentatively speculates on what such creations might look like. First he imagines us symbiotically merging with electronic components to form a world of cyborgs, using biological and artificial intelligence to sense and regulate the planet's state. Eventually he sees wet organic life having to be replaced by dry electronic life that is better able to cope with an over-hot Sun. He even imagines such artificial life forms 'evolving' much faster than us, because information is transferred much faster by electronics than by neurons.

This is a popular science book. Yet it does reveal something about how the author's remarkable mind works - making intuitive leaps between topics that most of us would not think to connect. One moment he is dwelling autobiographically on the cultural environment that fostered his own inventiveness, the next he is considering how invention accelerated the evolution of life. The leaps are so great that at times even Lovelock seems unsure where his argument is going. But this is strangely appropriate when the subject is the long-term future.

A Rough Ride to the Future is a more optimistic book than its two predecessors, although Lovelock still thinks that there will be a massive drop in human population - by means that remain unclear. What he seems to be doing, perhaps unwittingly, is creating a belief system to help us through the difficulties ahead. There is a sense here that Lovelock has been to the mountain top, looked over, and seen a promised land. Now almost 95, he may not get there with us, but he wishes humanity and Gaia an exciting future together.

Tim Lenton is professor of climate change and Earth system science at the University of Exeter, $U K$.

e-mail:t.m.lenton@exeter.ac.uk

\title{
The clamorous mind
}

\section{Susanne Ahmari reviews a personal and scientific journey through obsessive-compulsive disorder.}

$\mathrm{T}$ The adage that you can't judge a book by its cover fails with David Adam's The Man Who Couldn't Stop. The title is scrawled repeatedly in urgent letters, thrusting us into the mindset of someone suffering a near-constant barrage of intrusive thoughts - the manifestation of obsessive-compulsive disorder (OCD) that is the book's topic. In the pages that follow, Adam, editor of Nature's Editorials section, leads us on an insider's tour of the OCD brain, providing insight into the cultural and scientific evolution of how we view and treat a disorder that affects up to $3 \%$ of people worldwide.

Adam's book is equal parts historical journey, scientific discussion and stark personal memoir of his battle with often shocking thoughts that come out of nowhere, the symptom of OCD that he describes as "a snowflake that fell from the summer sky". A common example of such thoughts is the fear of harming someone else despite having no intent or desire to do so. For Adam, they took the form of fears that he had accidentally contracted HIV. Although his book pays homage to earlier works, including Judith Rapoport's iconic The Boy Who Couldn't Stop Washing (E. P. Dutton, 1989), it has a unique voice born of first-hand observations of Adam's own changing mental processes, and his expertise as a science writer.

Although his historical anecdotes are informative and entertaining, Adam is at his best when linking the evolution of his illness with possible scientific explanations. Current theories suggest that OCD stems from abnormal communication in the brain between cortical areas involved in higher-order cognition - such as reward-based decision-making - and basal ganglia structures important for selecting actions. Human studies suggest that genetic and environmental factors contribute to this disruption. However, despite intensive efforts, it has been difficult to nail down specific genetic culprits, and the role of environmental factors, such as childhood infection with streptococcus, is unclear

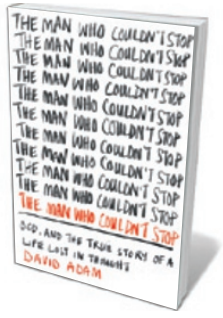

The Man Who Couldn't Stop:

OCD, and the True Story of a Life Lost in Thought DAVID ADAM

Picador: 2014 and controversial.

Accounts from sufferers may provide hints about how OCD evolves. In a particularly evocative story, Adam describes his attempts to combat the barrage of unwelcome thoughts about HIV by repeatedly calling the UK National AIDS hotline to check whether he was at risk. No matter how many times he called, disguising his voice to avoid recognition, he was only briefly reassured. He notes, "They would tell me that they had already given me an answer and that I needed to accept it. I didn't want that. I wanted the hit." This comparison between taking an addictive drug and seeking reassurance highlights important potential research avenues for neuroscientists trying to understand how inexorable cycles of obsessions and compulsions are initiated and maintained.

As Adam himself points out, the ending of the book is a little unsatisfying. There is no dramatic revelation of the root of his illness, no 'eureka' moment when scientists discover the cause of obsessions. His OCD gets significantly better with a lot of hard work, serotonin-reuptake inhibitors, exposure therapy with response prevention, and continuous vigilance, but intrusive thoughts linger in the background even

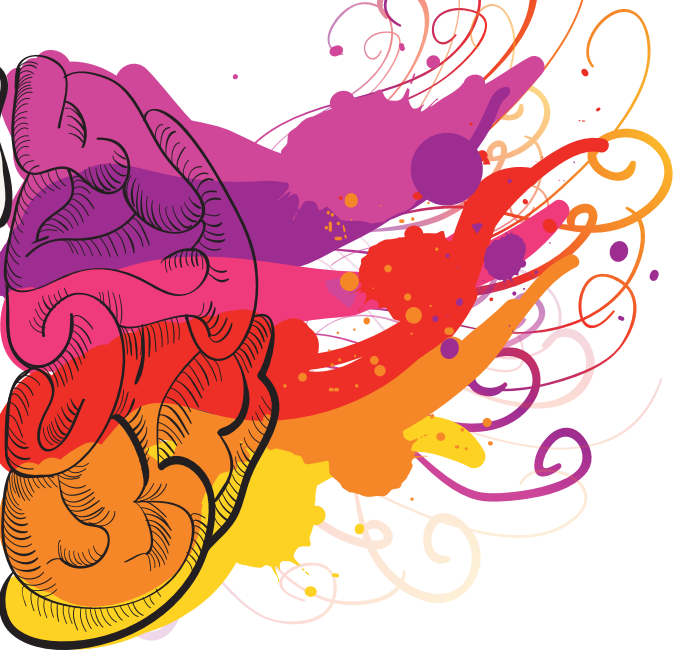


though he has received the best evidencebased OCD treatment. As he puts it, "it's a bit like being a recovering alcoholic. You are always a certain number of days past your most recent obsessive-compulsive episode."

Fortunately, misunderstanding about OCD, such as thinking that people with the condition are simply 'neat-freaks', is gradually decreasing. Adam makes an important contribution by increasing public awareness, and clarifying that OCD is not simply an exaggerated desire for hygiene and order, but rather a serious illness with many different symptoms caused by dysregulation of the brain's anxiety and cognitive-control circuits. He also helps to defuse the stigma associated with thoughts that many patients find shameful.

Another source of optimism is the abundance of innovative technologies that are being used to identify treatment targets for compulsive behaviours. For example, my laboratory uses miniature microscopes to peer inside the rodent brain and observe how neural activity changes as behaviours such as repetitive grooming develop. Such tools allow us "OCDis a bit to address longlike being $a$ standing quesrecovering tions, including alcoholic. You how compulsive are always a behaviours and certain number anxiety are related. of dayspast Although the yourmostrecent main readerepisode." ship of The Man Who Couldn't Stop is likely to be patients, families and clinicians, this book is also a valuable read for neuroscientists like me, who are searching for clues about the origins of OCD. There are some factual errors, such as the description of serotonergic neurotransmission, but this is a quibble compared with the book's contribution. People such as Adam hold in their brains some of the most valuable contributions to the fight against neuropsychiatric diseases: blow-by-blow accounts of how their obsessions, compulsions, thoughts and emotions are linked and evolve.

By partnering with patients, psychiatric researchers can extract testable hypotheses and recognize obsessions for what they are: not untouchable thoughts to be feared and locked up, but phenomena to be observed, understood and then let go like summer snowflakes melting in the sunlight.

Susanne Ahmari is director of the Translational OCD Laboratory at the University of Pittsburgh in Pennsylvania, where she uses neuroscience and clinical studies to seek brain abnormalities that lead to OCD.

e-mail:ahmarise@upmc.edu

\section{Books in brief}

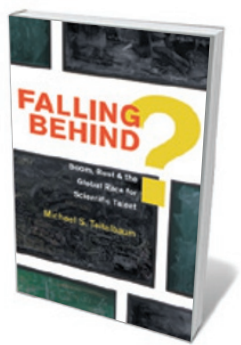

Falling Behind? Boom, Bust, and the Global Race for Scientific Talent

Michael S. Teitelbaum PRINCETON UNIVERSITY PRESS (2014)

Is US science and engineering really plagued by workforce shortages? It is not that simple, argues demographer Michael S. Teitelbaum in this analysis of US science policy. The evidence reveals a complex dynamic: since the 1950s, alarmism over 'falling behind' has driven five rounds of destabilizing boom and bust. The modi operandi of research universities and funding agencies are also problematic, as research funding, not market demand, controls supplies of young scientists and engineers. Lucid and convincing.

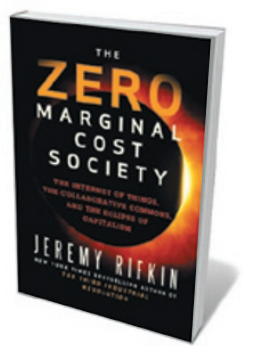

The Zero Marginal Cost Society: The Internet of Things, the Collaborative Commons, and the Eclipse of Capitalism Jeremy Rifkin PALGRAVE MACMILLAN (2014)

"The capitalist era is passing," proclaims social theorist Jeremy Rifkin in this manifesto for the collaborative commons, a new economic paradigm. "Ubiquitous computing" is spawning an Internet of Things, a digitized global infrastructure that will, he argues, pare marginal costs of production and distribution down to near zero and topple big profits. The thesis is admirable, but the ebullience can feel somewhat relentless as Rifkin rolls out a future of sustainable abundance, massive open online courses and crowd-funding.

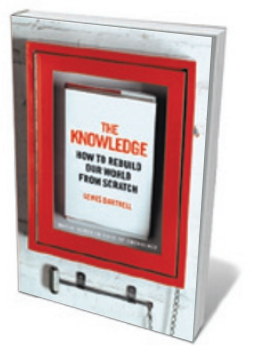

The Knowledge: How to Rebuild Our World from Scratch Lewis Dartnell BODLEY HEAD (2014)

Astrobiologist Lewis Dartnell offers the ultimate do-it-yourself guide to 'rebooting' human civilization after an asteroid impact, pandemic or nuclear war. With scientific nous, Dartnell depicts probable environmental scenarios on a stricken Earth and offers putative survivors instruction in the technologies needed to craft a culture from the ground up. Whether learning the basics on how to sow and grow a field of barley, melt and cast salvaged aluminium, build a windmill or craft a basic stethoscope, many will thrill to this reminder of our species' prodigious resilience.

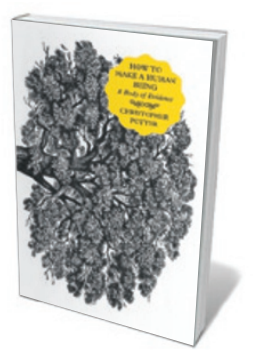

How to Make a Human Being: A Body of Evidence

Christopher Potter FOURTH ESTATE (2014)

In this follow-up to his much-vaunted You Are Here (HarperCollins, 2009), Christopher Potter illuminates the human in all its manifestations - from single cell to creator of culture. Finely judged quotes from scientific and literary luminaries such as John Archibald Wheeler and Marcel Proust alternate with erudite musings on our compatibility with the cosmos (backed by clear expositions of theoretical physics), human biology, neurology, culture, morality and religion. The scattershot narrative somehow coalesces into a brilliant whole - and a compelling case for anti-reductionism.

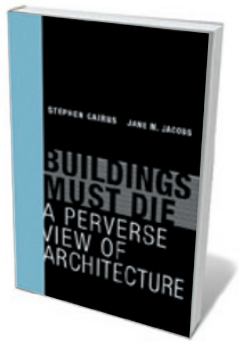

Buildings Must Die: A Perverse View of Architecture

Stephen Cairns and Jane M. Jacobs THE MIT PRESS (2014)

From the passage of time to the wrecking ball, the forces limiting a building's 'lifespan' are rife: all cities are potential ghost towns. Into this rubble-strewn terrain venture Stephen Cairns and Jane M. Jacobs, whose philosophical meditation on architectural death is enriched with materials science and thinking from the likes of architect Eero Saarinen. The evocative case studies range from the "ruin porn" of derelict Detroit in Michigan, to the weird morphology of Bangkok and its unfinished skyscrapers. Barbara Kiser 